\title{
A study of short term rotational variability in TNOs and Centaurs from Sierra Nevada Observatory
}

\author{
J. L. Ortiz ${ }^{1}$, P. J. Gutiérrez ${ }^{1,2}$, V. Casanova ${ }^{1}$, and A. Sota ${ }^{1}$ \\ 1 Instituto de Astrofísica de Andalucía, CSIC, Apt 3004, 18080 Granada, Spain \\ ${ }^{2}$ Laboratoire d'Astrophysique de Marseille, Traverse du Siphon, BP 8, 13376 Marseille Cedex 12, France
}

Received 9 April 2003 / Accepted 19 June 2003

\begin{abstract}
Broad band CCD observations focused on short-term rotational variability have been carried out for six TNOs: $1999 \mathrm{TD}_{10}, 1999 \mathrm{TC}_{36}, 2000 \mathrm{~EB}_{173}$, (20000) Varuna, (28978) Ixion, $2002 \mathrm{CR}_{46}$, and four Centaurs: $2000 \mathrm{QC}_{243}, 2001 \mathrm{PT}_{13}$, $2002 \mathrm{PN}_{34}$ and $2002 \mathrm{GO}_{9}$ using the $1.5 \mathrm{~m}$ telescope at Sierra Nevada Observatory (Granada, Spain) since mid 2001. Three of the bodies exhibit periodic double-peaked lightcurves with amplitudes larger than 0.4 mag while another four show periodic variability with amplitudes below $0.20 \mathrm{mag}$. Basic physical properties of these objects can be derived or constrained from the observations. Here we present a summary of the main results obtained for these objects.
\end{abstract}

Key words. minor planets, asteroids - Kuiper Belt

\section{Introduction}

Trans-Neptunian Objects (TNOs) and Centaurs are outer Solar System minor bodies from which Jupiter family comets originate. All these groups seem to share the same origin in the Kuiper or Edgeworth-Kuiper Belt (e.g. Fernández 1980; Duncan et al. 1988, etc.). At present, it is believed that these objects are among the least evolved in the Solar System. Therefore, the study of these bodies will help us in understanding the formation and evolution of the Solar System. Many of the current observational studies of TNOs and Centaurs are focused on color photometry or spectroscopy (e.g. Jewitt \& Luu 2001; Boehnhardt et al. 2002; Barucci et al. 2002; Hainaut \& Delsanti 2002; Doressoundiram et al. 2002; etc.). By using statistical analysis, most of these studies try to find likely correlations between color trends and orbital parameters.

Other works related to TNOs and Centaurs are focused on the study of their short-term variability (e.g. Davies et al. 1998; Hainaut et al. 2000; Gutiérrez et al. 2001; Farnham 2001; Ortiz et al. 2002; Sheppard \& Jewitt 2002; Bauer et al. 2002; Sekiguchi et al. 2002; Peixinho et al. 2002; etc.). These studies can give us hints on the shape, density and other basic physical properties of these bodies (e.g. Sheppard \& Jewitt 2002) as well as on the original size-shape distribution (Lacerda et al. 2003). Besides, short-term variability studies of the largest members of the Kuiper Belt are very important because some knowledge on the rotational state is needed in order to apply the appropriate thermophysical models to derive the albedos of these bodies. Also, accurate rotation periods can provide the

Send offprint requests to: J. L. Ortiz,

e-mail: ortiz@iaa.es link needed to check heterogeneity of surfaces as suggested by spectroscopy data (Barucci et al. 2002; Lazzarin et al. 2003).

However, time-resolved photometry requires considerable observing time devoted to each object and therefore it is hard to carry out due to the difficulty of getting long observing runs in large telescopes. Smaller telescopes can be used in longer runs, but restricted to sufficiently bright TNOs.

In 2001, we started a CCD photometry program devoted to studying the short-term variability of some of the brightest TNOs and Centaurs from Sierra Nevada observatory. This paper summarizes part of the results already obtained by the ongoing program.

\section{Observations and data reduction}

The observations were carried out by means of the Instituto de Astrofísica de Andalucía 1.5m telescope at Sierra Nevada Observatory, in Granada, Spain. One-week observing runs were allocated in August 2001, September 2001, October 2001 and February 2002, March 2002, May 2002 and August 2002, but weather or technical problems prevented us from observing in some of the nights of each run. The bodies observed are listed in Table 1 along with other relevant data. The dates with usable data are also listed in Table 1.

As Centaurs and TNOs have typical drift rates ranging from one to a few arcsecs per hour, we used the shortest exposure time possible in order not to get elongated images of either the object or the field stars (depending on whether the telescope is tracked at sidereal or nonsidereal rate respectively). An exposure time of $100 \mathrm{~s}$ was short enough to avoid noticeable trailing under the best foreseeable seeing conditions, but long enough 
Table 1. Dates and geometric data (range) of the objects observed.

\begin{tabular}{|c|c|c|c|c|c|c|}
\hline & Object & $r_{\mathrm{h}}(\mathrm{AU})$ & $\Delta(\mathrm{AU})$ & $\alpha(\operatorname{deg})$ & No of images & Observing dates \\
\hline \multicolumn{7}{|l|}{ TNOs } \\
\hline & $1999 \mathrm{TC}_{36}$ & 31.3 & $30.57-30.49$ & $1.30-1.14$ & 205 & 13, 15, 17-20, AUG. (2001) \\
\hline & $1999 \mathrm{TD}_{10}$ & 12.7 & $11.75-11.77$ & $0.86-1.10$ & 450 & 23-26, OCT. (2001) \\
\hline & $2000 \mathrm{~EB}_{173}$ & 29.6 & $29.18-28.71$ & $1.72-0.87$ & 503 & 9-10, FEB. (2002); 8-10, MAR. (2002) \\
\hline & $\left(2000 \mathrm{WR}_{106}\right)$ Varuna & 43.1 & $42.33-43.34$ & $0.81-0.83$ & 343 & 8-9, FEB. (2002) \\
\hline & $\left(2001 \mathrm{KX}_{76}\right)$ Ixion & 43.1 & $42.81-42.87$ & $1.30-1.32$ & 180 & 15, 17-19, AUG. (2001) \\
\hline & $2002 \mathrm{CR}_{46}$ & 18.1 & $17.37-17.40$ & $2.15-2.23$ & 190 & 8-10, MAR. (2002) \\
\hline \multicolumn{7}{|l|}{ Centaurs } \\
\hline & $2000 \mathrm{QC}_{243}$ & 19.2 & $18.21-18.17$ & $1.12-0.77$ & 290 & 13, 15, 17-20, AUG. (2001) \\
\hline & $2001 \mathrm{PT}_{13}$ & 8.9 & $7.93-8.45$ & $2.67-5.89$ & 681 & 10-11, 13-14, SEP. (2001); 23-28, ОCT. (2001) \\
\hline & $2002 \mathrm{PN}_{34}$ & 13.4 & $12.50-12.52$ & $2.30-2.43$ & 260 & 28-30, AUG. (2002) \\
\hline & $2002 \mathrm{GO}_{9}$ & 14.4 & $13.17-13.18$ & $2.15-2.26$ & 263 & 18-19, MAY (2002) \\
\hline
\end{tabular}

so that the sky background was the dominating noise source. In order to get a good enough signal to noise ratio, most of the observations were obtained with no filter. Therefore, unless otherwise noted, the observations consisted of sequences of $100 \mathrm{~s}$ integrations with no filter. The number of images obtained is therefore very high, in the order of a few thousands. The typical seeing during the observations ranged from 1.1 arcsec to 2.5 arcsec, with median around 1.5 arcsec. A fast readout CCD was used in order not to spend large fractions of the nights reading out the CCD. The CCD chip format is $1024 \times 1024$ pixels and the total field of view is 7 arcmin $\times 7$ arcmin. The images were bias subtracted in the standard way and flat-fielded using a flatfield frame (the median of a large set of dithered twilight images of the sky at blank fields). No cosmic ray removal algorithms were used and we simply rejected the few images in which a cosmic ray hit was close to the objects. Relative photometry using seven field stars was carried out by means of Daophot routines. The synthetic aperture used was typically 8 to 12 pixels in diameter (the smallest possible in order to get the highest signal to noise). Care was taken not to introduce spurious signals of faint background stars or galaxies in the aperture. In cases where the TNOs or Centaurs were close to faint stars or galaxies, the data were rejected.

Since the TNOs and Centaurs move relatively slowly, we were able to use the same field stars within each observing run. The typical error bars of the individual $100 \mathrm{~s}$ integrations are 0.15 mag for the faintest targets we observed, and around 0.06 mag for the brightest ones. Both figures were considerably improved by averaging or "median averaging" the large amount of relative photometry data points. The approach of averaging is similar to using longer integrations times, but has the advantage that no trails are present in the images, comic ray hits are fewer and images are also less smeared.

In order to make absolute flux calibrations, one of the field stars was calibrated against Landolt standards close in airmass so that extinction corrections were negligible. For the absolute calibrations the diameter of the aperture used was large enough for both the Landolt star and the field star so that no flux was lost. Due to the fact that the observations were carried out in a non standard photometric band, the absolute calibration of the data has a large uncertainty which could reach up to $0.5 \mathrm{mag}$ in some cases. This has been indicated with the words "approximate $V$ magnitude" in the plots presented here. The absolute calibration is irrelevant for this investigation, in which short-term variability of the object is obtained by using the stars in the field of view.

The time-resolved observations were inspected for periodicities by means of the Lomb technique (Lomb 1976) as implemented in Press et al. (1992). Typical $1 \sigma$ errors in the period determination for our typical four-day observing windows are in the order of $0.02 \mathrm{~h}$ (neglecting the interfering effects of the aliases). In the cases in which we were able to use longer time frames, the period determination is more precise. The reference stars were also inspected for short term variability but none was found.

\section{Results}

The main photometric results are listed by object and summarized in Table 2.

(20000) Varuna (formerly $2000 W R_{106}$ ). The short-term variability of this very large TNO has been studied by several authors. Farnham (2001) found a $3.17 \mathrm{~h} \pm 0.01 \mathrm{~h}$ periodicity and Jewitt \& Sheppard (2002) reported a double-peaked lightcurve with a period of $6.3442 \pm 0.0002 \mathrm{~h}$, consistent with the previous observations. Our own data, taken in coordination with the IRAM-30 m radiotelescope in order to obtain a new derivation of Varuna's albedo (Lellouch et al. 2002) are shown in Fig. 1. The periodogram analysis of our data showed a clear periodicity peak that implied a rotation period of $6.35 \pm 0.02 \mathrm{~h}$, which was consistent with the rotation period given by Jewitt \& Sheppard 2002 (within error bars). By combining our data with those published in Jewitt \& Sheppard (2002) (after correcting our data for light travel time, heliocentric, geocentric distances, and phase angle) and performing a periodogram analysis we find that the peak with the highest spectral power corresponds to a rotation period of $6.3436 \mathrm{~h} \pm 0.0001 \mathrm{~h}$. The quality of the rotational phase curve is illustrated in the second plot of Fig. 1. Although the 6.3436-h rotation period is the one with the highest spectral power, other possible periods (which differ by less than $2 \%$ in spectral power) yield phase curves that are acceptable by visual inspection. These correspond to $6.3319 \mathrm{~h}, 6.3554 \mathrm{~h}$, and $6.3177 \mathrm{~h}$. However, a period of $6.3442 \mathrm{~h} \pm 0.0002 \mathrm{~h}$ as reported by Jewitt \& Sheppard (2002) 
Table 2. Summary of the photometric results.

\begin{tabular}{|c|c|c|c|}
\hline & Object & $\begin{array}{c}\text { Amplitude } \\
\text { (mag) }\end{array}$ & $\begin{array}{l}\text { Photometric } \\
\text { period (hrs) }\end{array}$ \\
\hline \multicolumn{4}{|l|}{ TNOs } \\
\hline & $1999 \mathrm{TD}_{10}$ & $0.65 \pm 0.05$ & $7.71 \pm 0.02$ \\
\hline & $1999 \mathrm{TC}_{36}$ & $0.06 ?$ & Several possibilities? \\
\hline & $2000 \mathrm{~EB}_{173}$ & $<0.1$ & $6.75^{c} \pm 0.01$ \\
\hline & Varuna & $0.41 \pm 0.02$ & $3.1718^{a} \pm 0.0001$ \\
\hline & Ixion & $<0.15$ & None detected \\
\hline & $2002 \mathrm{CR}_{46}$ & $<0.15$ & $3.66^{b}$ or $4.35^{b} \pm 0.02$ \\
\hline \multicolumn{4}{|l|}{ Centaurs } \\
\hline & $2000 \mathrm{QC}_{243}$ & $0.75 \pm 0.09$ & $4.57 \pm 0.02$ \\
\hline & $2001 \mathrm{PT}_{13}$ & $0.16 \pm 0.02$ & $4.1546 \pm 0.0001$ \\
\hline & $2002 \mathrm{PN}_{34}$ & $0.18 \pm 0.04$ & 4.23 or $5.11 \pm 0.03$ \\
\hline & $2002 \mathrm{GO}_{9}$ & $0.14 \pm 0.04$ & 6.97 or $9.67 \pm 0.04$ \\
\hline
\end{tabular}

${ }^{a}$ Combining our data with those by Jewitt \& Sheppard (2002).

${ }^{b}$ Confidence level below $50 \%$.

${ }^{c}$ Possible alternate periods are $6.68 \mathrm{~h}$ and $6.82 \mathrm{~h}$.
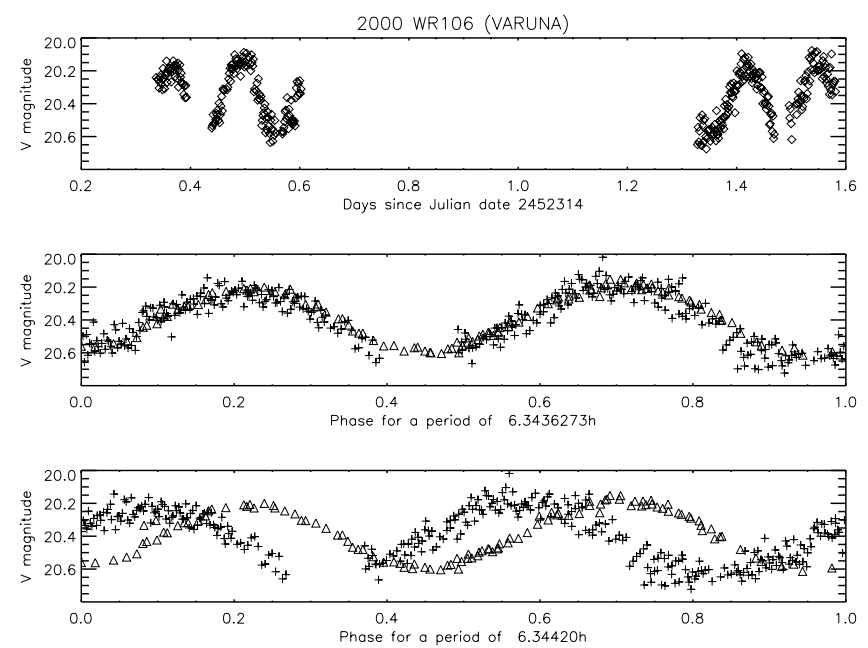

Fig. 1. Upper panel: Varuna's magnitude versus time (days) from our observatios. Middle panel: our data and Jewitt \& Sheppard's (2002) data phased to the period we propose. Our data are shown as plus signs and Jewitt \& Sheppard's (2002) are shown as triangles. Lower panel: our data and Jewitt \& Sheppard's (2002) data phased to the period proposed by Jewitt \& Sheppard (2002). Our data are shown as plus signs and Jewitt \& Sheppard's (2002) are shown as triangles. As can be seen, the data are not in phase, which implies that $6.3442 \mathrm{~h}$ is incorrect.

is not compatible with our data because the phase plot is unacceptable (see lower panel of Fig. 1). The amplitude of our lightcurve is $0.41 \pm 0.09 \mathrm{mag}$, entirely consistent with that derived by Jewitt \& Sheppard (2002).

$1999 T D_{10}$. This object was studied by Consolmagno et al. (2000) prior to our observations. They reported that a large amplitude periodic variation was detected for this body, with a period of $5.8 \mathrm{~h}$. The analysis of our data yields a peak at $7.71 \pm 0.02 \mathrm{~h}$, and an alias with somewhat lower spectral power is clearly seen at $5.8 \mathrm{~h}$. The rotational phase plots from our data show that a period of $7.71 \pm 0.02 \mathrm{~h}$ is reasonable whereas $5.8 \mathrm{~h}$ is inconsistent (Fig. 2). The amplitude of our lightcurve is close
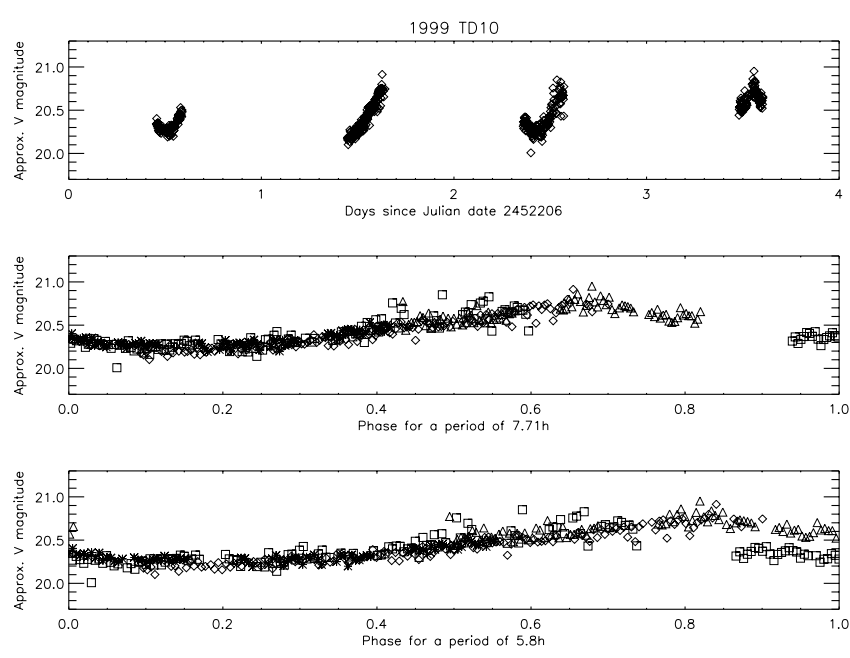

Fig. 2. Upper panel: $1999 \mathrm{TD}_{10}$ magnitude versus time (days). Lower panels: rotational phase curves for two possible periods, $7.71 \mathrm{~h}$ and $5.8 \mathrm{~h}$, respectively. As can be seen, a period of $5.8 \mathrm{~h}$ does not yield an acceptable phase curve. Different symbols correspond to different dates.

to $0.65 \pm 0.05 \mathrm{mag}$, which is the value reported by Consolmagno et al. (2000). The period could in principle be refined in a similar fashion as is done here with Varuna, by combining our data with the previous observations if they were available. Two other very recent and independent works (Choi et al. 2002 and Rousselot et al. 2002) agree with our period determination within $0.02 \mathrm{~h}$. Therefore one can safely rule out the $5.8 \mathrm{~h}$ periodicity and adopt $7.71 \pm 0.02 \mathrm{~h}$ as the correct one. We cannot yet determine whether the lightcuve is single-peaked (i.e. the rotation period would be $7.71 \pm 0.02 \mathrm{~h}$ ) or double-peaked (i.e. the actual spin period would be $2 \times 7.71 \mathrm{~h}=15.42 \pm 0.04 \mathrm{~h}$ ). Nevertheless, it is very likely that indeed $15.42 \mathrm{~h}$ corresponds to the true rotation period because an albedo asymmetry causing such a large amplitude lightcurve is hard to imagine (see discussion section).

$2001 P T_{13}$. For this object we have a large data set that has already been published by Ortiz et al. (2002). In that paper, the two main data sets were analyzed separately. We have now been able to improve the period determination in Ortiz et al. (2002), by combining the two separate data sets that were reported in that paper. Once light travel time, geocentric and heliocentric distances are properly accounted for, we found a refined period of $4.15456 \pm 0.00005 \mathrm{~h}$ which likely corresponds to a rotation period of $8.3091 \mathrm{~h} \pm 0.0001 \mathrm{~h}$. Figure 3 shows the lightcurve and rotational phase curve of this object. Farnham (2001) derived a period of $8.4 \mathrm{~h}$ for this object which would be in agreement with our derivation if one assumes a $0.1 \mathrm{~h}$ uncertainty in his determination. From the periodogram, other marginally acceptable periods are $8.3391 \mathrm{~h}$ and $8.2424 \mathrm{~h}$.

$2000 Q C_{243}$. This body shows a large amplitude oscillation with a periodicity of $4.57 \pm 0.02 \mathrm{~h}$, which likely corresponds to a rotation period of $9.14 \pm 0.04 \mathrm{~h}$ because the lightcurve appears to be double-peaked. Therefore, this body also appears to be a very irregular one, like $1999 \mathrm{TD}_{10}$. The rotational phase curve for a period of $9.14 \mathrm{~h}$ is shown in Fig. 4. 

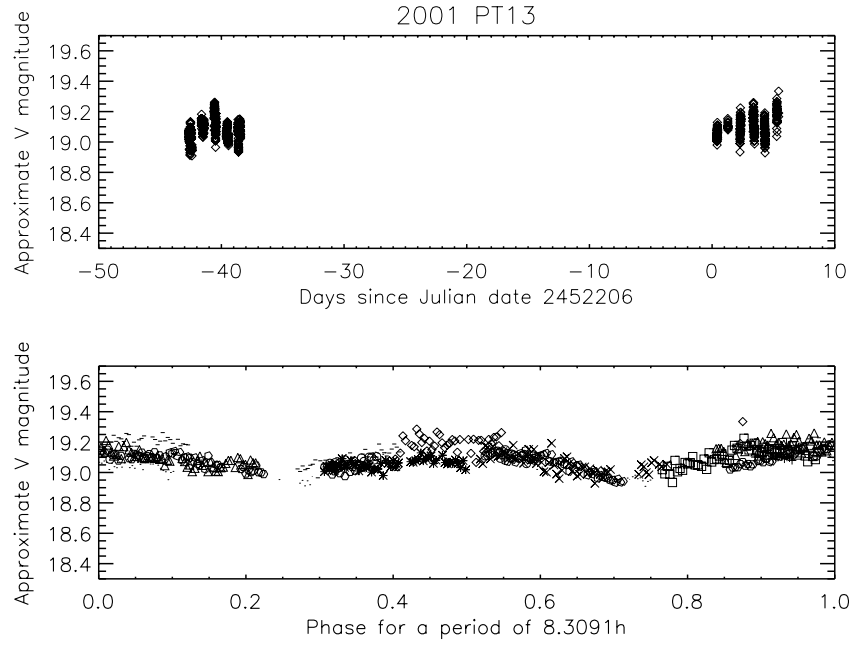

Fig. 3. Upper panel: $2001 \mathrm{PT}_{13}$ 's magnitude versus time (days). Lower panel: rotational phase curves for a possible spin period of $8.3091 \mathrm{~h}$. Different symbols correspond to different dates.
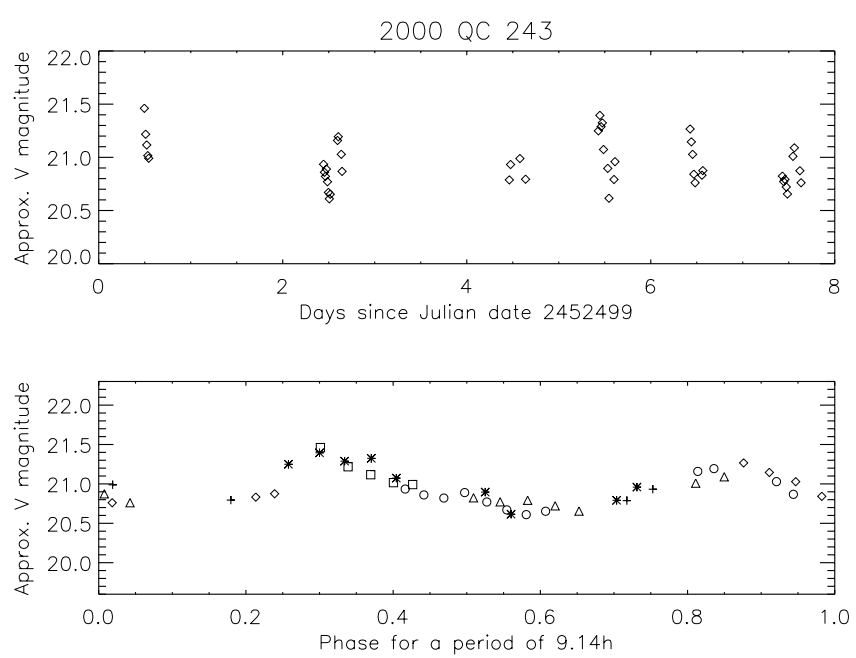

Fig. 4. Upper panel: $2000 \mathrm{QC}_{243}$ 's magnitude versus time (days). Lower panel: rotational phase curve for a period of $9.14 \mathrm{~h}$. Different symbols correspond to different dates.

$1999 T C_{36}$. Our data for this body are somewhat puzzling to us. From the September 2001 run we determined a very low amplitude (0.06 mag) periodic signal of $6.21 \pm 0.02 \mathrm{~h}$ with a high confidence level. Nevertheless, we have not been able to confirm it with prior data taken in August 2001 (Ortiz et al. 2001) nor in a subsequent run, in which we obtained inconsistent periodicities. Since this object has been found to be binary (Trujillo \& Brown 2001) the lightcurve may be complex or perhaps our data are simply not good enough. Peixinho et al. (2002) studied its short-term variability but found none within the precision of their measurements.

$2002 C R_{46}$. The analysis of the data shows a possible periodicity of $4.35 \pm 0.04 \mathrm{~h}$ and also of $3.66 \pm 0.04 \mathrm{~h}$ (an alias of very similar spectral power), but the confidence level is below $50 \%$. Hence, our analysis is inconclusive regarding a rotational period. The amplitude is lower than $0.15 \mathrm{mag}$.

$2000 E B_{173}$. By combining the two data sets of this object in February and March 2002, a period of $6.75 \pm 0.01 \mathrm{~h}$ has
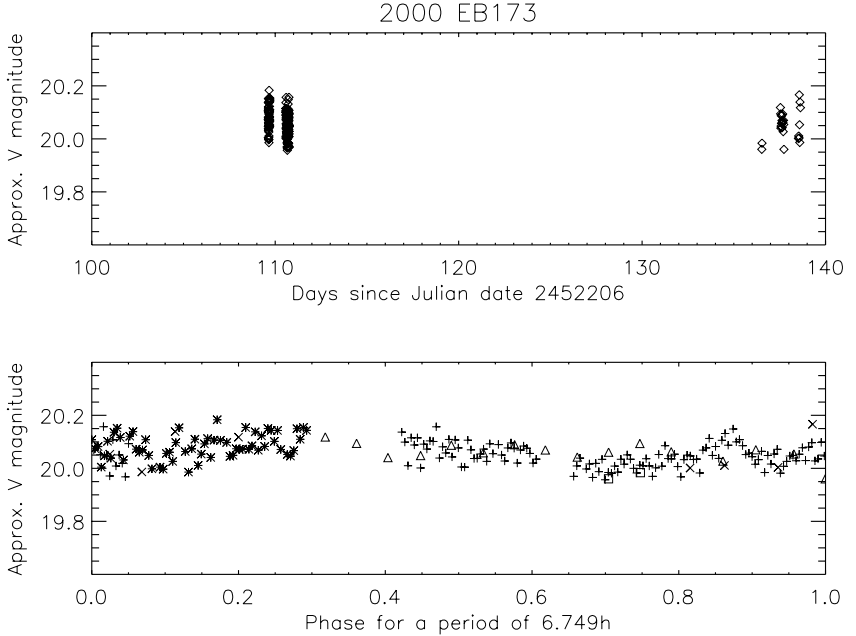

Fig. 5. Upper panel: $2000 \mathrm{~EB}_{173}$ 's magnitude versus time (days). Lower panel: rotational phase curve for one possible period. The different symbols correspond to different dates.

been found at a high and confident spectral power (98\%). The periodogram also shows two peaks of somewhat lower (but still similar) power at $6.82 \pm 0.01 \mathrm{~h}$ and $6.68 \pm 0.01 \mathrm{~h}$. The peak to peak amplitude of the oscillation is lower than $0.1 \mathrm{mag}$ (Fig. 5). Schaefer \& Rabinowitz (2002) obtained photometric measurements of this object on 78 days and concluded that rotational modulation, if present, should have a peak-to-peak amplitude below 0.097. This is consistent with our determination. Sheppard \& Jewitt (2002) observed this object in four days and did not detect measurable photometric variations within a 0.15 mag range. This is also consistent with our results. We cannot yet stablish whether the lightcurve is single-peaked or double-peaked. The small amplitude could be due to the fact that the object is nearly spherical or that it was observed nearly pole on.

(28978) Ixion (formerly $2001 K X_{76}$ ). The data we obtained for this body in August 2001 are still inconclusive regarding a possible rotation period. At least we can say that if this body has a periodic variation of brightness, it is below 0.15 mag or the period is very long.

$2002 P_{34}$. The periodogram analysis of the data shows two peaks at $4.23 \pm 0.03 \mathrm{~h}$ and a possible alias at $5.11 \pm 0.03 \mathrm{~h}$ with similar spectral power and with very high confidence levels $(99.85 \%$ and $99.75 \%$, respectively). The lightcurve seems to be double-peaked, and therefore, $8.45 \pm 0.06 \mathrm{~h}$ or $10.22 \pm 0.06 \mathrm{~h}$ could be likely spin periods. The rotational phase curves for these spin periods are shown in Fig. 6. Both rotational curves are acceptable, although the rotational curve for $10.22 \mathrm{~h}$ looks somewhat better than the curve for $8.45 \mathrm{~h}$. Therefore, we cannot reliably establish which is the actual spin period. The amplitude is below 0.20 mag.

$2002 \mathrm{GO}_{9}$. Like the previous one, the periodogram of the data shows two very confident peaks ( $99.9 \%$ confidence) at $6.97 \pm 0.03 \mathrm{~h}$ and $9.67 \pm 0.03 \mathrm{~h}$. Both rotational phase curves (Fig. 7) are acceptable and we cannot yet stablish which is the actual spin period. A simple sinusoidal fit of the rotational phase indicates a mean amplitude of $0.14 \pm 0.04 \mathrm{mag}$. The curve 

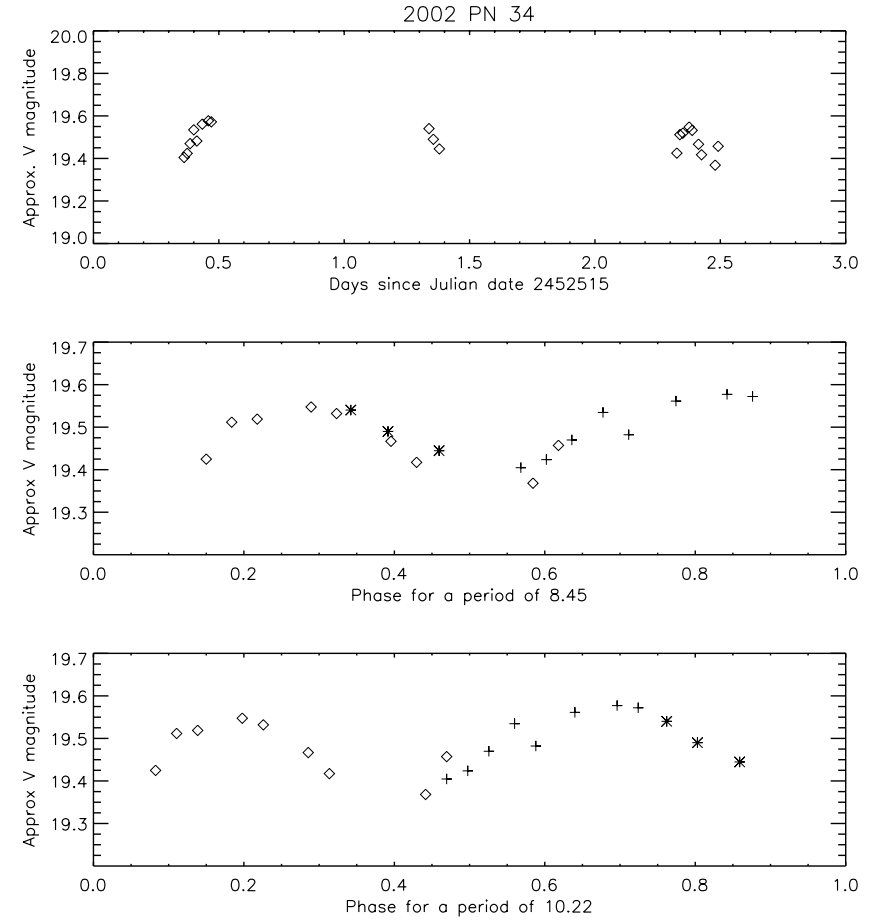

Fig. 6. Upper panel: $2000 \mathrm{PN}_{34}$ 's magnitude versus time (days). Lower panels: rotational phase curves for two possible periods, $8.45 \mathrm{~h}$ and $10.22 \mathrm{~h}$. Different symbols correspond to different dates.

seems to be single-peaked, but a double-peaked curve cannot be ruled out.

\section{Discussion}

Following Burns \& Safronov (1973), rotational excitation damping times are usually assumed to be much less than the age of the solar system. A more recent study on ellipsoids (Molina et al. 2003) points out that damping times can be even shorter if one takes into account the energy dissipation due to internal stresses. Thus, it is expected that most of the Kuiper Belt objects must be in pure rotation around their principal axes of maximal moment of inertia. Under that premise, and assuming that they are single-body systems, the short-term variability in these objects is due to their shapes and/or to their albedo and topographic variations on the surface. If they were binary systems, mutual eclipses would also modulate the brightness variability produced by their rotation. So far, among the known KBOs (more than 500), less than 10 are known to be binary systems. A study by Noll et al. (2002) points out that only $4 \pm 2 \%$ of the TNOs are binaries. In our list, only $1999 \mathrm{TC}_{36}$ has been cataloged as binary. Concerning the other objects of this study, most of them have also been observed by several groups in order to determine their colors or spectral characteristic and no evidences pointing out that they are binary system have been reported. Therefore, we can assume that, apart from $1999 \mathrm{TC}_{36}$, the variability observed in these objects is due to their shape and/or to albedo and topographic variation on their surfaces.

In general, double-peaked lightcurves are attributed to the rotation of an irregularly shaped body while single-peaked curves are attributed to albedo variations on the surface.
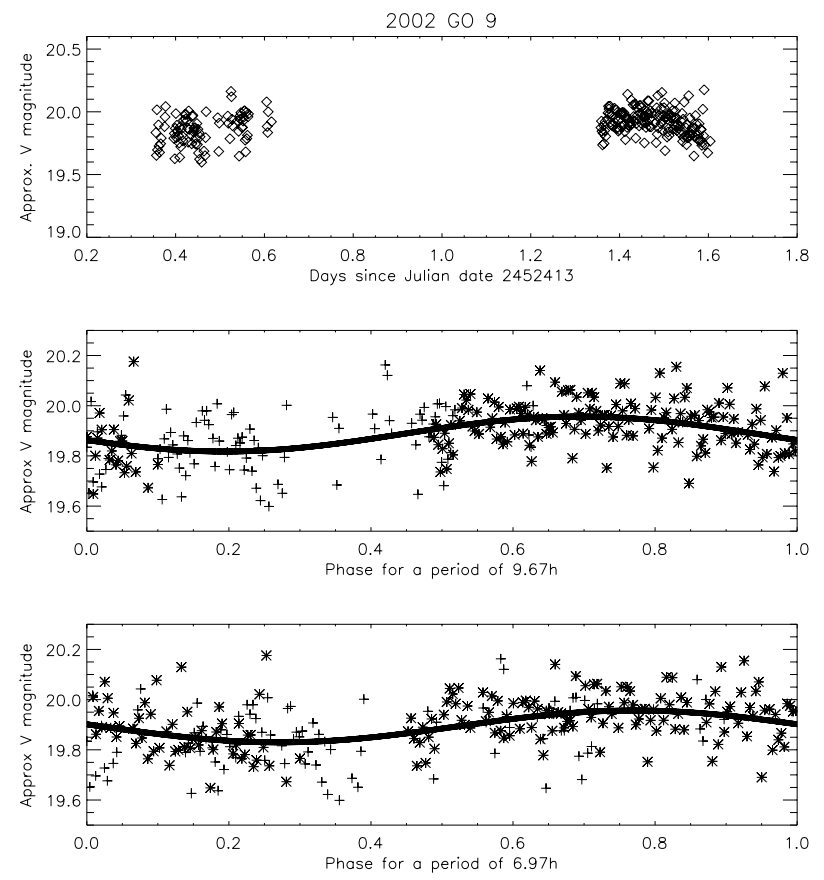

Fig. 7. Upper panel: $2002 \mathrm{GO}_{9}$ 's magnitude versus time (days). Lower panels: rotational phase curves for two possible periods, $9.67 \mathrm{~h}$ and $6.97 \mathrm{~h}$. Different symbols correspond to different dates. The line is a sinusoidal fit to the data.

Actually, as was demonstrated by Russell (1906), it is not possible to distinguish the effect of irregular shape from the effect of the albedo with a single color light curve. The appropriate albedo distribution could lead to a double-peaked lightcurve. So far, the only method to reliably distinguish shape-induced from albedo-induced brightness variations is simultaneous observations in the visible and in the thermal infrared. Also, timeresolved color measurements can help. Albedo-induced brightness variations are frequently accompanied by color variations along the rotation period, whereas shape-induced variations usually are not (e.g. Jewitt \& Sheppard 2002). We do not have color information and therefore we cannot reliably conclude which is the final reason for the observed brightness changes.

Taking the results of other minor bodies observations into account, albedo variegations frequently produce smaller brightness changes than the shaped-induced ones. Typical short-term variability amplitudes due to albedo are smaller than $0.15 \mathrm{mag}$. From our list of ten observed bodies, three of them (Varuna, $1999 \mathrm{TD}_{10}$ and $2000 \mathrm{QC}_{243}$ ) show short-term variability with larger amplitudes than $0.40 \mathrm{mag}$, and in principle, these bodies could be irregularly shaped ones.

In an extensive study, Jewitt \& Sheppard (2002) discuss about the possible causes for the variability observed in Varuna. From their study, they conclude that, given its large size, Varuna may be a rotationally distorted rubble pile with a likely density around $1000 \mathrm{~kg} / \mathrm{m}^{3}$.

$1999 \mathrm{TD}_{10}$ has a mean diameter around $100 \mathrm{Km}$ (Choi et al. 2002; Consolmagno et al. 2000). Given its relatively small size, this object could be a collisional fragment with irregular shape (Davis \& Farinella 1997). If the variability is due to irregular shape, from the amplitude of its lightcurve, the minimum 
axial ratio is 1.8:1.0. Following the calculations by Davidsson (2001), if this body is assumed to be prolate with no internal tensile strength, the minimum density required to avoid spontaneous rotational breakup is $315 \mathrm{~kg} / \mathrm{m}^{3}$ or $80 \mathrm{~kg} / \mathrm{m}^{3}$ provided that the body is rotating with a period of $7.71 \mathrm{~h}$ or $15.42 \mathrm{~h}$, respectively. If the body is spherical, the corresponding limits for the density are $183 \mathrm{~kg} / \mathrm{m}^{3}$ and $45 \mathrm{~kg} / \mathrm{m}^{3}$, respectively. On the other hand, if this body had a density similar to the density estimated for Varuna $\left(1000 \mathrm{~kg} / \mathrm{m}^{3}\right)$, its rotational breakup critical period would be approximately $4.3 \mathrm{~h}$ (if it is prolate) or $3.3 \mathrm{~h}$ (if it is spherical), which are well below the actual rotation period. Nevertheless, it is important to note that, according to the expressions given by Davidsson (2001) and for that density, a minimum material strength of $4 \mathrm{kPa}$ would be necessary in order to resist shear fracture due to the rotation (this minimum is calculated for a spherical body rotating with $15.42 \mathrm{~h}$ ). For that density, a lower material strength would indicate that the body is structurally damaged.

Assuming a 0.04 geometric albedo, $2000 \mathrm{QC}_{243}$ has a mean diameter of $200 \mathrm{Km}$ (Ortiz et al. 2002). According to Davis \& Farinella (1997) and given its size, collisions could have transformed this body into rubble pile. Assuming that this centaur is a strengthless prolate body, with an axis ratio of 1.9:1.0 (derived from the lightcurve amplitude), its critical density to avoid rotational breakup would be around $300 \mathrm{~kg} / \mathrm{m}^{3}$ if the spin period is $9.14 \mathrm{~h}$. For a typical density of $1000 \mathrm{~kg} / \mathrm{m}^{3}$, the critical period would be $4.45 \mathrm{~h}$ (assuming prolate shape). Therefore this body would be mechanically stable if it is rotating with a period of $9.14 \mathrm{~h}$. On the contrary, if this body is spherical, rotating with a period of $4.57 \mathrm{~h}$, the lower limit for the density to avoid rotational breakup would be around $500 \mathrm{~kg} / \mathrm{m}^{3}$. Nevertheless, even for the typical density of $1000 \mathrm{~kg} / \mathrm{m}^{3}$, its critical period would be $3.3 \mathrm{~h}$, which is very close to $4.57 \mathrm{~h}$, and a large material strength would be necessary in order to stay intact.

In our list, four objects show rather confident short-term variability with amplitudes smaller than 0.20 mag. They are $2000 \mathrm{~EB}_{173}, 2001 \mathrm{PT}_{13}, 2002 \mathrm{PN}_{34}$ and $2002 \mathrm{GO}_{9}$. In principle, it cannot be reliably concluded whether these objects are spherical or not, although some of them show clear doublepeaked curves. The size of these bodies can be approximately estimated from their absolute magnitudes. Assuming a geometric albedo of 0.05 , their diameters would be, approximately, $670 \mathrm{Km}, 100 \mathrm{Km}, 160 \mathrm{Km}$, and $100 \mathrm{Km}$, respectively. If $2000 \mathrm{~EB}_{173}$ is a spherical body, rotating with a period of $6.75 \mathrm{~h}$ and assuming a null internal strength, the minimum density required to avoid rotational breakup is $239.2 \mathrm{~kg} / \mathrm{m}^{3}$, which is well below the typical density of $1000 \mathrm{~kg} / \mathrm{m}^{3}$. Nevertheless, even for such a low density, according to the expressions given by Davidsson (2001), a material strength of $93 \mathrm{kPa}$ is necessary in order to withstand shear fracturing, and stay intact. For the typical density of $1000 \mathrm{~kg} / \mathrm{m}^{3}$, the corresponding material strength is $1000 \mathrm{kPa}$. If the actual period were $13.5 \mathrm{~h}$, the corresponding material strength would be $234 \mathrm{kPa}$. These values are considerably large, and several orders of magnitude larger than the expected values for cometary internal strength (much lower than $1 \mathrm{kPa}$ ) (e.g. Greenberg et al. 1995; Asphaug \& Benz 1996). Therefore, if this body were spherical and were rotating with a period of $6.75 \mathrm{~h}$ it would likely be structurally damaged. In this respect, and in long term time scales, fractured regions could be displaced, distorting the spherical body. Actually, this body could be an irregular rubble pile, formed by shear fractures due to rotation, and, therefore, similar to Varuna. The relative small amplitude of its lightcurve could be a result of being observed nearly pole on.

For the other three bodies, $2001 \mathrm{PT}_{13}, 2002 \mathrm{PN}_{34}$ and $2002 \mathrm{GO}_{9}$, similar considerations as the ones given above result in some constraints that are compiled in Table 3 . The corresponding values for the material strength in order to withstand shear fracturing are also very large. Therefore, it is likely that these bodies are structurally damaged. In this respect, such structurally damaged bodies might even be a potential source for small-size bodies with no need for collisions.

Finally, we have not detected reliable short-term variability in Ixion, nor in $2002 \mathrm{CR}_{46}$ and therefore cannot give constraints on their shape, density and internal structure.

Sheppard \& Jewitt (2002) presented a systematic investigation of rotational light curves of large TNOs. From their own study and by compiling other works on variability, they found that $32 \%$ of the sample displays significant light curve amplitudes (larger than $0.15 \mathrm{mag}$ ). We warn however, that compiling reports on variability from the literature may be somewhat misleading, because some investigators may report just the objects for which they have found variability and not mention the objects for which they found no variability. This would artificially increase the percentage of objects that show variability.

Our own independent statistics based on the 10 objects observed would indicate that $40 \%$ of the objects had variations larger than $0.15 \mathrm{mag}$ and $30 \%$ of them showed larger variations than $0.4 \mathrm{mag}$, although our statistics includes not only TNOs, but also Centaurs which do not meet the criteria imposed by Sheppard \& Jewitt (2002) that $\mathrm{H}$ must be equal or less than 7.5.

If we add our results on TNOs with $\mathrm{H}$ smaller than 7.5 to the compilation by Sheppard \& Jewitt, their statistics is hardly modified. Now seven of 24 objects (29\%) would show variability above $0.15 \mathrm{mag}$ and $21 \%$ would show variability above 0.4 mag. Including the objects in Ortiz et al. (2003), the percentages decrease to $26 \%$ and $18 \%$ respectively.

Concerning possible statistics for Centaurs alone, based on our results and other studies, such as the one by Davies et al. (1998), the equivalent figures to the TNO case are $40 \%$ of objects (4/10) showing variability above $0.15 \mathrm{mag}$ and $10 \%(1 / 10)$ of objects show variability above $0.4 \mathrm{mag}$. Nevertheless we must stress that the population is too small to draw meaningful conclusions yet.

\section{Summary and conclusions}

From our list of 10 objects, significant short-term variations have been detected in 3 objects. Another four objects show reliable variability with amplitude smaller than $0.20 \mathrm{mag}$. The other three objects do not show reliable variability within the 0.15 mag range. In general, assuming no internal strength, these bodies require a small lower limit for the density $\left(<300 \mathrm{~kg} / \mathrm{m}^{3}\right)$ in order to be rotationally stable again spontaneous breakup. Nevertheless, given the size of the objects in 
Table 3. Characteristics and properties of the bodies showing short-term variability. The material strength of the last column corresponds to the minimum material strength necessary to withstand shear fracture and stay intact.

\begin{tabular}{|c|c|c|c|c|c|c|c|c|}
\hline \multirow[t]{2}{*}{ Object } & \multirow[t]{2}{*}{$\begin{array}{c}\text { Diameter } \\
(\mathrm{km})\end{array}$} & \multirow[t]{2}{*}{$\begin{array}{l}\text { Minimum } \\
\text { axis } \\
\text { ratio }\end{array}$} & \multirow[t]{2}{*}{$\begin{array}{l}\text { Spin } \\
\text { period } \\
\text { (h) }\end{array}$} & \multicolumn{2}{|c|}{$\begin{array}{c}\text { Minimum } \\
\text { density } \\
\text { to avoid breakup }\left(\mathrm{kg} / \mathrm{m}^{3}\right)\end{array}$} & \multicolumn{2}{|c|}{$\begin{array}{c}\text { Critical } \\
\text { period (h) } \\
\rho=1000 \mathrm{~kg} / \mathrm{m}^{3}\end{array}$} & \multirow[t]{2}{*}{$\begin{array}{c}\text { Minimum } \\
\text { material strength }(\mathrm{kPa}) \\
\text { for } \rho=1000 \mathrm{~kg} / \mathrm{m}^{3}\end{array}$} \\
\hline & & & & Prolate & Spherical & Prolate & Spherical & \\
\hline \multirow[t]{2}{*}{$1999 \mathrm{TD}_{10}$} & $100^{1}$ & $1.8: 1$ & 7.71 & 315 & 183 & 4.3 & 3.3 & \\
\hline & & & 15.42 & 80 & 45 & & & 4 \\
\hline \multirow[t]{2}{*}{$2000 \mathrm{QC}_{243}$} & $200^{2}$ & $1.9: 1$ & 9.14 & 300 & & 4.5 & & \\
\hline & & & 4.57 & & 500 & & 3.3 & 2300 \\
\hline $2000 \mathrm{~EB}_{173}$ & $670^{3}$ & $<1.1: 1$ & 6.75 & & 240 & & & 1000 \\
\hline $2001 \mathrm{PT}_{13}$ & $100^{3}$ & $1.16: 1$ & 8.30 & & 160 & & 3.3 & 13 \\
\hline \multirow[t]{2}{*}{$2002 \mathrm{PN}_{34}$} & $160^{3}$ & $1.14: 1$ & 10.22 & & 104 & & 3.3 & 23 \\
\hline & & & 8.45 & & 152 & & & \\
\hline \multirow[t]{2}{*}{$2002 \mathrm{GO}_{9}$} & $100^{3}$ & $1.14: 1$ & 9.67 & & 116 & & 3.3 & 10 \\
\hline & & & 6.97 & & 224 & & & \\
\hline
\end{tabular}

${ }^{1}$ Choi et al. (2002); Consolmagno et al. (2000).

${ }^{2}$ Ortiz et al. (2002).

${ }^{3}$ Estimated from its absolute magnitude assuming an albedo of 0.05 .

this study and their relatively fast spin periods, a large material strength (at least 2 or 3 orders of magnitude larger than the expected cometary material strength) is required in order to stay intact and to resist shear fracturing, even for low densities. Therefore, if TNOs and Centaurs do not have high material strengths they will be structurally damaged by rotation. These bodies could be fractured merely due to rotation, even if they were not affected by collisions.

Acknowledgements. We thank H. Boehnhardt for his constructive and helpful comments. We are also grateful to the Sierra Nevada Observatory staff. This research was based on data obtained at the Observatorio de Sierra Nevada which is operated by the Instituto de Astrofísica de Andalucía, CSIC. This work was supported by contracts PNE-001/2000-C-01, AYA-2001-1177 and AYA-2002-0382. European FEDER funds for these contracts are also acknowledged.

\section{References}

Asphaug, E., \& Benz, W. 1996, Icarus, 121, 225

Bauer, J. M., Meech, K. J., Fernández, Y. R., et al. 2002, PASP, 114, 1309

Barucci, M. A., Boehnhardt, H., Dotto, E., et al. 2002, A\&A, 392, 335

Boehnhardt, H., Delsanti, A., Barucci, A., et al. 2002, A\&A, 395, 297

Burns, J. A., \& Safronov, V. S. 1973, MNRAS, 165, 403

Consolmagno, G. J., Tegler S. C., Rettig, T., \& Romanishin, W. 2000 , Bull. Amer. Astron. Soc., 32, \# 21.07

Choi, Y. J., Prialnik, D., \& Brosch, N. 2002, in Asteroids, Comets, Meteors Conference, Berlin

Davidsson, B. J. R. 2001, Icarus, 149, 375

Davis, D. R., \& Farinella, P. 1997, Icarus, 125, 50

Davies, J. K., McBride, N., Ellison, S. L., Green, S. F., \& Ballantyne, D. R. 1998, Icarus, 134, 213

Doressoundiram, A., Peixinho, N., \& de Bergh, C. 2002, AJ, 124, 2279

Duncan, M., Quinn, T., \& Tremaine, S. 1988, ApJ, 328, L69

Farnham, T. L. 2001, Bull. Amer. Astron. Soc., 33, \#12.10

Fernández, J. A. 1980, MNRAS, 192, 481
Greenberg, J. M., Mizutani, H., \& Yamamoto, T. 1995, A\&A, 295, L35

Gutiérrez, P. J., Ortiz, J. L., Alexandrino, E., Roos-Serote, M., \& Doressoundiram, A. 2001, A\&A, 371, L1

Hainaut, O. R., Delahodde, C. E., Boehnhardt, H., et al. 2000, A\&A, 356,1076

Hainaut, O. R., \& Delsanti, A. C. 2002, A\&A, 389, 641

Jewitt, D. C., \& Luu, J. 2001, AJ, 122, 2099

Jewitt, D. C., \& Sheppard, S. S. 2002, AJ, 123, 2110

Lazzarin, M., Barucci, M. A., Boehnhardt, H., Tozzi, G. P., \& De Bergh., C. 2003, AJ, 125, 1554

Lellouch, E., Moreno, R., Ortiz, J. L. et al. 2002, A\&A, 391, 1133

Lacerda, P., \& Luu, J. 2003, Icarus, 161, 174

Landolt, A. U. 1992, AJ, 104, 340

Lomb, N. R. 1976, Astroph. Space Sci., 39, 447

Noll, K., Stephens, D., Grundy, W. et al. 2002, Bull. Amer. Astron. Soc. 34, 09.05

Molina, A., Moreno, F., \& Martínez-López, F. 2003, A\&A, 398, 809

Ortiz, J. L., Lopez-Moreno, J. J., Gutiérrez, P. J., \& Baumont, S. 2001, Bull. Amer. Astron. Soc., 33, \#12.11

Ortiz, J. L., Baumont, S., Gutiérrez, P. J., \& Roos-Serote, M. 2002, A\&A, 388, 661

Ortiz, J. L., Casanova, V., \& Sota, A. 2003, Presentation at the First decadal review of the Edgeworth-Kuiper Belt. Towards New Frontiers, 11-15 March 2003, Antofagasta, Chile

Peixinho, N., Doressoundiram, A., \& Romon-Martin, J. 2002, New Astron., 7, 359

Press, W. H., Teukolsky, S. A., Vetterling, W. T., \& Flannery B. P. 1992, in Numerical Recipes in Fortran: the art of scientific computing, 2nd edn. (London: Cambridge Univ. Press), 569

Rousselot, P., Poulet, F., \& Petit, J.-M. 2002, Asteroids, Comets, Meteors Conference, 16-08, Berlin

Russell, H. 1906, ApJ, 24, 1

Schaefer, B. E., \& Rabinowitz, D. L. 2002, Icarus, 160, 52

Sekiguchi, T., Boehnhardt, H., Hainaut, O. R., \& Delahodde, C. E. 2002, A\&A, 385, 281

Shepppard, S., \& Jewitt, D. C. 2002, AJ, 124, 1757

Trujillo, C. A., \& Brown, M. E. 2001, IAUC, 7787 